\title{
Recovery of Flagellar Inner-arm Dynein and the Fertilization Tubule in Chlamydomonas ida5 Mutant by Transformation with Actin Genes
}

\author{
Akio Ohara*, Takako Kato-Minoura ${ }^{\S}$, Ritsu Kamiya*, \& , and Masafumi Hirono*đ \\ *Department of Biological Sciences, Graduate School of Science, University of Tokyo, Tokyo 113-0033, Japan, \\ and ${ }^{\S}$ National Institute for Basic Biology, Okazaki 444-8585, Japan
}

Key words: dynein mutant/flagellar actin/actin-like protein/chimeric actin gene/fertilization tubule

\begin{abstract}
The ida5 mutant of Chlamydomonas, first isolated as a mutant lacking a subset of axonemal inner-arm dyneins, has recently been shown to lack conventional actin owing to a serious mutation in its gene. It lacks inner-arm dyneins probably because actin is an essential subunit for their assembly. In addition, male gametes of ida5 are unable to produce the fertilization tubule, a structure that contains a core of actin filament bundles. To establish that those observed deficiencies are solely attributable to the loss of actin, and to provide a basis for future studies on the actin function in this organism, we examined in this study whether transformation of this mutant with cloned actin genes can rescue the mutant phenotypes. Cotransformation of the double mutant ida5arg 2 with the wild-type actin gene and arginino-succinate lyase gene that suppresses the arg 2 mutation yielded several transformants that displayed increased motility. All of them were found to have acquired the introduced actin gene in the genome and the product actin in the flagella, and regained the missing inner-arm dyneins and wild-type motility. In addition, most transformants also became able to grow the fertilization tubule when mating reaction was induced. In addition to the wild-type actin gene, we also used a chimeric actin gene in which the $\mathrm{N}$-terminal 12 amino-acid sequence of Chlamydomonas actin was replaced by that of the greatly divergent Tetrahymena actin. Transformants with this gene also resulted in recovery of inner-arm dynein and 70-80\% of the wild-type level of motility. These results established that the lack of inner-arm dynein and the fertilization tubule in $i d a 5$ are consequences of its loss of conventional actin. Furthermore, they demonstrate that Chlamydomonas offers an excellent experimental system with which to study the structure-function relationship of actin by means of mutant analysis.
\end{abstract}

Actin is a subunit of inner-arm dynein in cilia and flagella of various organisms $(13,18,23,24)$. In Chlamydomonas, six out of the total of seven inner-arm dynein subspecies have been found to contain actin (7). A recent study in our laboratory has shown that a Chlamydomonas dynein-deficient mutant, ida5, has a mutation in the conventional actin gene such that its product is totally lost (10). This mutant lacks four inner-arm dynein subspecies and swims more slowly than wild type (9). In addition, its gametes display low mating efficiency because the male gametes cannot produce the fertilization tubule, an apical process that contains a core of actin filament bundles. Rather unexpectedly, however, the growth rate and the manner of cytokinesis in ida5 do not differ from those in wild type. Since Chlamydomonas has only one gene of conventional ac-

Correspondence to: Dr. Masafumi Hirono, Department of Biological Sciences (Zoological Institute), Graduate School of Science, University of Tokyo, Hongo, Bunkyo-ku, Tokyo 113-0033, Japan.

Tel: +81-3-3812-2111 ex. 4429, Fax: +81-3-3816-1965

E-mail: hirono@biol.s.u-tokyo.ac.jp tin (28), this suggests a striking possibility that Chlamydomonas can grow normally without conventional actin. Also, it is somewhat puzzling that only four inner-arm subspecies are lost in this mutant, while actin is contained in six inner-arm subspecies in wild type. However, on close examination, two of the three inner-arm subspecies remaining in ida 5 have been found to contain a novel actin-like protein instead of conventional actin (10). This protein, named NAP (Novel Actin-like Protein), has recently been found to be identical with a putative actin-related protein, of which the DNA sequence has been reported to have $64 \%$ identity with the conventional actin (15; Kato et al., manuscript in preparation). NAP may be able to substitute for actin in some, but not all, cellular functions.

The availability of a null mutant of conventional actin gene makes Chlamydomonas an excellent organism with which to study the structure-function relationship of conventional actin by means of mutant analysis. To establish that the observed mutant phenotypes of ida5 are solely attributable to the loss of conventional actin, and to provide a basis for the future studies using site- 
directed mutagenesis, here we examined whether the above deficiencies in ida5 can be rescued by transformation with the wild-type actin gene. We also wanted to evaluate the extent of rescue, if they are rescued at all. In addition, we tested a modified actin gene for transformation as a model of experiment with mutated actin genes.

\section{MATERIALS AND METHODS}

\section{Strains and Cell Culture}

Chlamydomonas reinhardtii $137 \mathrm{c}$ (wild type), ida5 (9) and arg2 (27) were used. Cells were grown in the TAP medium (4) supplemented with $50 \mathrm{mg} / 1$ of arginine when required. For gametogenesis, cells were incubated in nitrogen-free medium (5) for 4-6 hours.

\section{Plasmids}

The plasmid pARG7.8 carrying the arginino-succinate lyase (ASL) gene (1) was used as a selectable marker for transformation of the double mutant ida5arg2. pA7, a plasmid containing the cloned wild-type actin gene (Fig. 1) (28), was used for the rescue experiment.

A plasmid containing chimeric actin gene in which the $\mathrm{N}$ terminal sequence of Chlamydomonas actin was replaced by that of Tetrahymena actin was constructed as follows. A $2 \mathrm{~kb}$ EcoRI-NotI fragment of pA7 containing 5 region of actin gene was subcloned into the vector pBluescript $\mathrm{KS}(-)$ (Stratagene Inc., La Jolla, CA). Using this plasmid (pAO1) as a template, polymerase chain reaction was performed with primers DN1 (5'-GCGGATCCCATTTTGAATCCTGCGT G-3') and DN2 (5'-GCGGATCCGGTGAGCAGGTGTTCA GA-3') to amplify almost the entire sequence of pAO1 except for the region corresponding to the $\mathrm{N}$-terminal 12 aminoacids of actin. The PCR product was digested at BamHI sites within both primer sequences (underlined) and ligated to make a plasmid pAO1 $\Delta$ N12. At the same time, a short sequence for the N-terminal 12 amino-acids of Tetrahymena actin was amplified by PCR using a plasmid pTpActin (6) as a template and using primers NT1 (5'-GCGGATCCGACAGT GACTCACCCGCT-3') and NT2 (5'-CCGGATCCATACCT GAACCGTTATCG-3'). The PCR product was also digested at $\mathrm{BamHI}$ sites at primer sequences (underlined) and inserted into the $B a m \mathrm{HI}$ site in pAO $1 \Delta \mathrm{N} 12$. The sequence of the $\mathrm{N}$ terminal region in the constructed plasmid (pOA2) was confirmed by DNA sequencing. The deduced amino-acid sequence from the confirmed sequence was MGSDSDSPAIVI DNGSGMDPAGF. The amino acids derived from Tetrahymena sequence are underlined, and artificial sequence caused by compiling are shown in italic. The EcoRI-NotI restriction fragment of pA7 was replaced by the EcoRI-NotI restriction fragment of pOA2. This plasmid was named pA7/Tet $12 \mathrm{Chl}$ (Fig. 1) and used for transformation.

\section{Transformation}

The wild-type (pA7) or chimeric actin gene (pA7/Tet12Chl) was introduced into ida5 cells by cotransformation with ASL gene (pARG7.8) as a selectable marker. All plasmid DNAs used for transformation had been linealized by digestion with HindIII (pA7 and pA7/Tet12Chl) or BamHI (pARG7.8). The double mutant ida5arg2 was grown in the TAP medium supplemented with arginine, treated with autolysin (5) for 1 hour, and transformed by the glass bead procedure of Kindle (11). For each transformation, a mixture of $1 \mu \mathrm{g}$ of pARG7.8 with $1 \mu \mathrm{g}$ of $\mathrm{pA} 7$ or $\mathrm{pA} 7 / \mathrm{Tet} 12 \mathrm{Chl}$ was used. After transformation, cells were spread onto TAP agar plates without arginine supplement and incubated for 7 days under constant illumination. Colonies on the plates were isolated and grown in the liquid TAP medium. The swimming speed of each clone was checked under a microscope and only those transformants that displayed improved motility were used for further studies.

\section{Southern blot analysis}

Chlamydomonas genomic DNA was purified by the method described in (32). The DNA of each clone was digested with $P v u I I$, loaded on a $1 \%$ agarose gel, and transferred on to a Hybond- $\mathrm{N}^{+}$membrane (Amersham International, Buckingham, UK). The membrane was processed for hybridization with $P v u I I-H i n d I I I$ fragments of pA7 insert as a probe (Fig. 1). The probes were labeled using DIG DNA labeling kit (Boehringer Mannheim Biochemica, Mannheim, Germany). Hybridizing bands were detected using DIG luminescent detection kit (Boehringer Mannheim Biochemica).

\section{Isolation of Axoneme and Dynein}

Flagella were detached from the cell body using dibucaine and purified by differential centrifugation (33). Dynein was obtained by extracting the demembranated flagellar axoneme with a buffer solution containing $0.6 \mathrm{M} \mathrm{KCl}$, and fractionated by chromatography on a Mono Q column (Pharmacia LKB Biotechnology), as described previously (7).

\section{Electrophoresis}

Whole cell lysate or axoneme was analyzed by SDS-polyacrylamide gel electrophoresis (SDS-PAGE) (14). For two-dimensional gel electrophoresis, isoelectric focusing was performed using Immobiline Dry Strip gel (Pharmacia LKB Biotechnology) and followed by SDS-PAGE in the second direction as described previously (10). The composition of dynein heavy chains was analyzed by SDS-PAGE with a 3-5\% acrylamide gradient and a 3-8 M urea gradient (22).

\section{Western blot analysis}

Samples of whole cells or axonemes were analyzed according to Towbin et al. (31). Antisera raised against oligo-peptides of the N-terminal sequences of Chlamydomonas actin and Tetrahymena actin $(6,28)$ were affinity-purified $(20)$ using fusion proteins expressed in $E$. coli. Immunoreactive bands were de- 
tected using alkaline phosphatase-conjugated secondary antibody (Cappel Research Products, Durham, NC) and BCIP /NBT Phosphatase Substrate System (Kirkegaard and Perry Laboratories, Gaithersburg, MD).

\section{Motility assessment}

The swimming velocities of Chlamydomonas cells were measured by tracing swimming paths using a video recording system connected to a dark-field microscope. All measurements were carried out at $25^{\circ} \mathrm{C}$.

\section{Electron microscopy}

Axonemes were centrifuged and the pellet was fixed with $2 \%$ glutaraldehyde in the presence of $1 \%$ tannic acid. Specimens were post-fixed with $1 \% \mathrm{OsO}_{4}$, block stained with $1 \%$ uranyl acetate, dehydrated in a series of ethanol solutions, embedded in Epon 812, and thin-sectioned. Sections were doublestained with uranyl acetate and lead citrate.

\section{Observation of fertilization tubules}

Plus mating type gametes of wild type, ida5 or transformants were incubated in the presence of $15 \mathrm{mM}$ dibutyryl-cAMP and $1 \mathrm{mM}$ IBMX for 1 hour to induce fertilization tubule growth (21). The cells were then fixed with $3.6 \%$ formaldehyde and permeabilized with $0.05 \%$ NP-40. BODIPY-phallacidin (Molecular Probes, Eugene, OR) was added to the fixed cells to a final concentration of 1-2 unit/ml, and incubated for 1 hour in the dark. Cells precipitated on the bottom of a tube were resuspended in Perma-Fluor (Immunon, Pittsburgh, PA) and observed with a fluorescence micro-

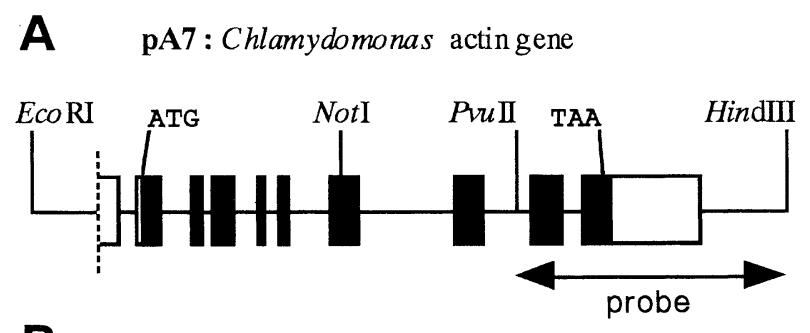

B pA7/Tet12Chl : Chimeric actin gene

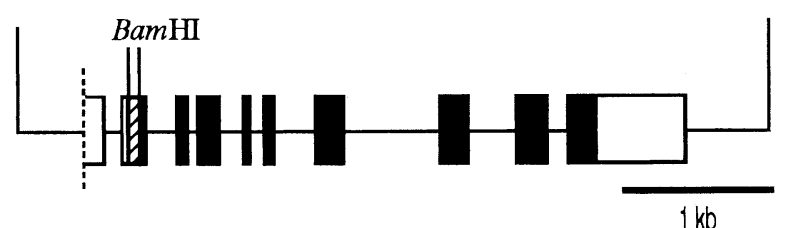

Fig. 1. Structures of the Chlamydomonas (pA7) and Tetrahymena /Chlamydomonas chimeric actin genes (pA7/Tet12Chl). Solid and open boxes indicate coding and non-coding exons, respectively. The lines represent introns and flanking sequences. The 5 -end of the first exon is shown by a dotted line because the start point of transcription has not been determined. The region of Tetrahymena actin sequence in the chimeric actin gene is indicated by shaded box. The arrow represents the probe used for the Southern blot analysis. scope (Axioplan, Carl Zeiss, Inc., Thornwood, NY). The fluorescent images were acquired with a chilled-CCD camera and an ARGUS20 image processor (Hamamatsu Photonics, Hamamatsu, Japan).

\section{RESULTS}

Transformation of ida5 cells with the wild-type and the chimeric actin genes

For transformation of the ida5 cells with the wild-type actin gene, the plasmid pA7 (Fig. 1A) was introduced into the double mutant ida5arg2 by means of cotransformation with the ASL gene which complements the arg2 mutation. The glass bead method of Kindle (11) was used for transformation. Colonies formed on the selective plates were transferred to liquid media and their motility was examined under a microscope. About $50 \%$ of randomly chosen clones exhibited clearly improved motility as compared with the mother strain. Of those clones with improved motility, six clones from each case were saved and analyzed further.

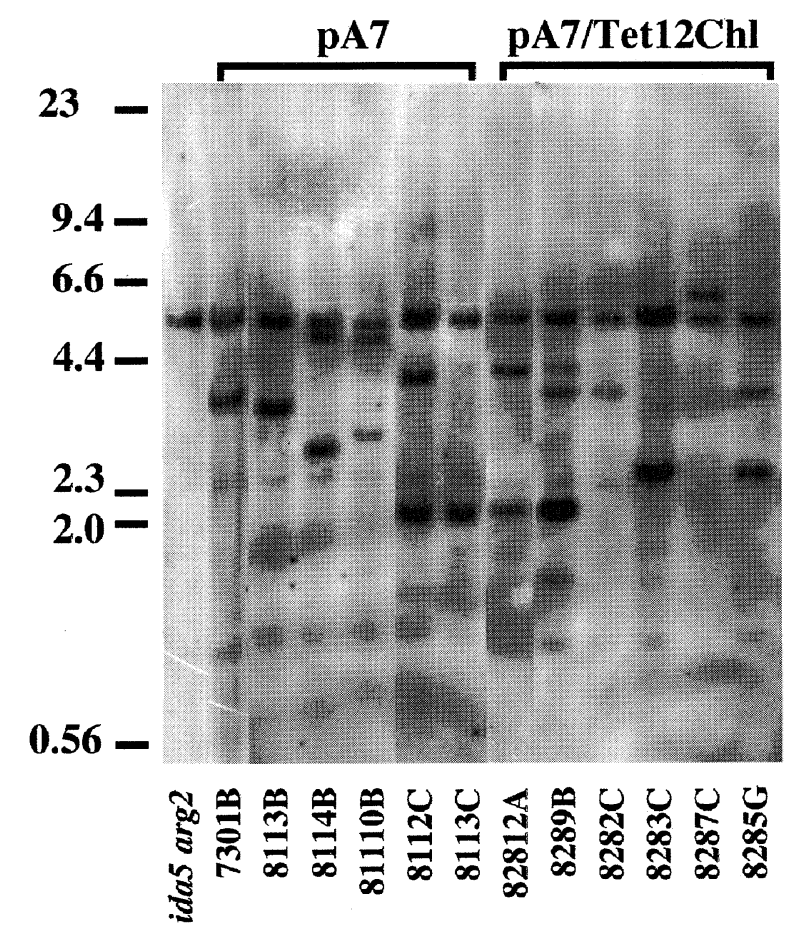

Fig. 2. Southern blot analysis showing the exogenous actin sequence(s) in the genome of transformants. The genomic DNA isolated from the parent strain (ida5arg2) and the transformants were digested with $P v u \mathrm{II}$. The probe used was $P v u \mathrm{II}-H i n \mathrm{dIII}$ fragments of pA7 insert (see Fig. 1). The transformants 7301B through $8113 \mathrm{C}$ are those transformed with pA7, while $82812 \mathrm{~A}$ through $8285 \mathrm{G}$ are those transformed with $\mathrm{pA} 7 / \mathrm{Tet} 12 \mathrm{Chl}$. The $5.5 \mathrm{~kb}$ band originating from the endogenous actin gene is indicated by an arrow. 
In addition to the wild-type actin gene, we also tested a chimeric actin gene, of which the N-terminal 12 amino-acid sequence has been replaced by the Tetrahymena actin sequence. The reason for using this actin gene is that the $\mathrm{N}$-terminal sequence of actin has been shown to be important for its function such as the interaction with myosin (29). Furthermore, the Tetrahymena actin has only about $75 \%$ homology to conventional actin and has been shown to display several unusual features (6); in fact, only three amino acids are shared by Chlamydomonas and Tetrahymena actins in the 12 amino-acid $\mathrm{N}$-terminal sequence. Transformation with the chimeric actin gene (pA7/Tet12Chl, Fig. 1B), performed essentially the same way as above, also resulted in clearly improved motility in about $50 \%$ of randomly chosen clones.

To see if the exogenous actin gene(s) has actually been introduced in the genome of these clones, the genomic DNA from each clone was analyzed by Southern hybridization using a fragment of the cloned genomic actin gene as a probe (Fig. 2). Since ida5 has an actin gene sequence with a one-base deletion, a $5.5 \mathrm{~kb}$ band representing the endogenous actin gene was detected in all the genomic DNAs examined. In addition, all the transformants displayed one or more additional bands, indicating that at least one copy of exogenous actin gene was incorporated.

Previous studies have shown that nuclear transformation in Chlamydomonas mostly takes place through a process of non-homologous recombination $(2,11$, 17). Thus it was expected that the exogenous actin genes were incorporated into sites different from the original actin gene locus and, therefore, genetic cross of the actin-transformants with the parent strain arg2 may yield daughter cells that display the slow-swimming phenotype of ida5. Experiments with two representative transformants $(8112 \mathrm{C}$ and $8113 \mathrm{C}$ ) in fact produced daughter cells with the $i d a 5$ phenotype, indicating that the actin gene was incorporated into a site independent of the original actin locus.

\section{Actin and NAP in the Transformants}

We next examined if the actin genes incorporated into the transformants actually produce actin. Western blot analyses of the whole cell lysate from these transformants, using an antibody specific to Chlamydomonas actin, indicated that actin was indeed expressed but its amount varied greatly from one transformant to another (Fig. 3A). In each case, the expressed amount was lower than that in the wild-type cells. With the six chimeric transformants, on the other hand, Western analysis using an antibody specific to the N-terminal se-
(A)

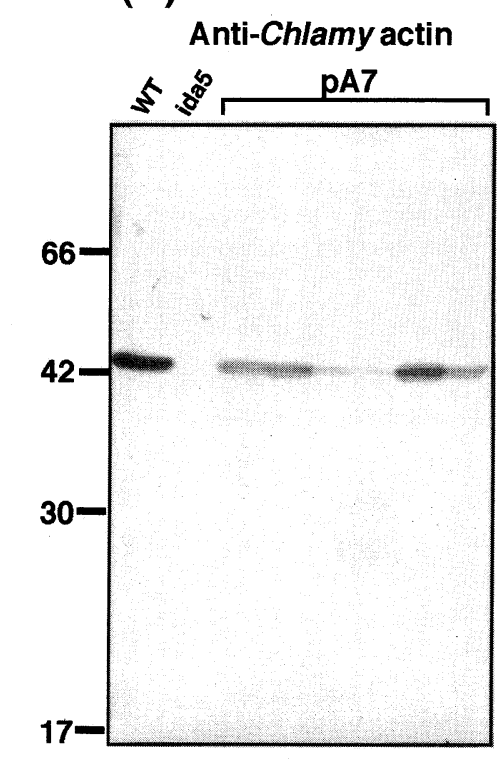

(kDa)
(B)
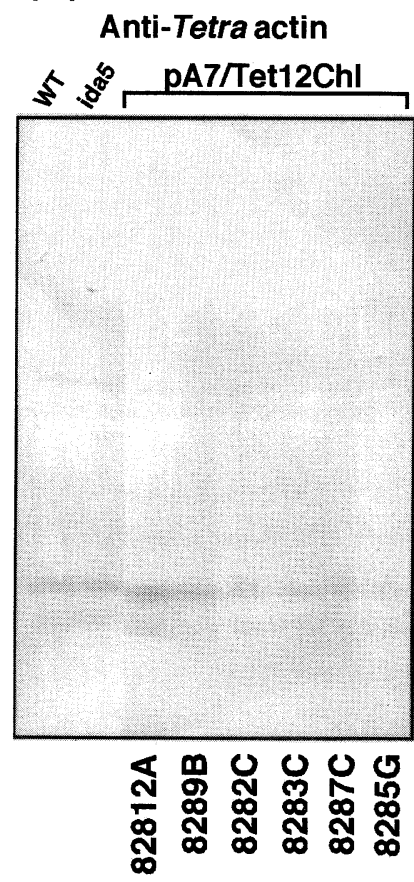

(C)

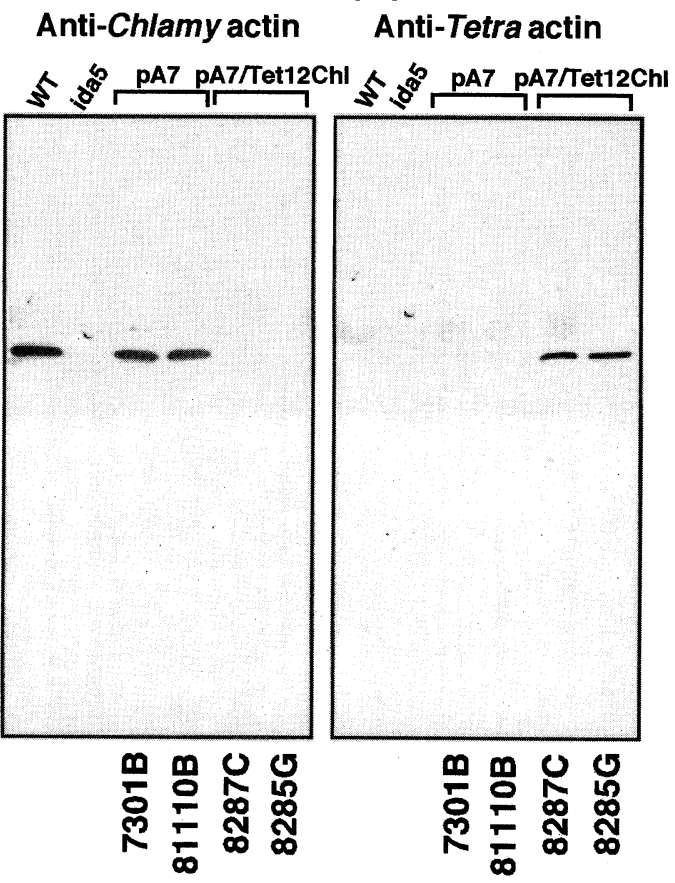

Fig. 3. Western blot analysis of the transformants. Whole cell lysates (A and B) or axonemes (C and D) of transformants were electrophoresed, transferred to membrane, and probed with anti-Chlamydomonas actin antibody (A and C) or with an antibody specific to the N-terminal sequence of Tetrahymena actin (B and D). A: wild-type transformants, B: chimeric transformants. C and D: axonemes of two transformants from each transformation group were isolated and analyzed. Bars with numbers indicate positions of molecular mass standards. 
quence of Tetrahymena actin that was included in the chimeric actin sequence did not detect any positive band (Fig. 3B). Thus the expression of the chimeric actin, if any, must be extremely low.

Although the actin expression in the chimeric transformants was undetectable by Western analysis on the whole cell lysate, the improved motility of those transformants suggested that they have recovered inner dynein arms, probably because actin is produced in a small amount and incorporated into flagella. We examined this possibility by performing Western analysis on the axonemes of transformants. As shown in Fig. 3C and $\mathrm{D}$, each antibody cross-reacted with a band of about $43 \mathrm{kDa}$ in the axoneme from the corresponding transformants, i.e., anti-Chlamydomonas antibody reacted with transformants with the wild-type gene, and anti-Tetrahymena antibody reacted with the transformants with the chimeric gene. Hence, both wild-type and chimeric genes introduced into ida5arg2 cells must express their products. Chimeric actin was detected in the axoneme and not in the cell lysates probably because the small amount of actin is preferentially localized in
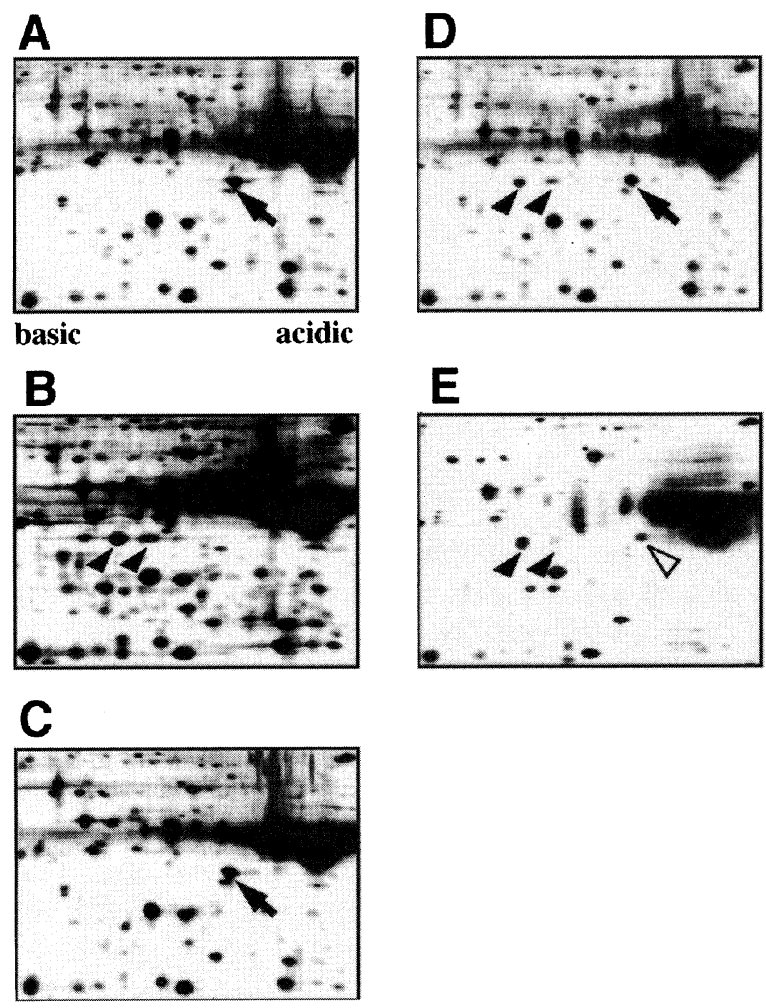

Fig. 4. Two-dimensional gel electrophoresis patterns of axoneme. Only the area containing the spots of actin and NAP are shown. A: wild type, B: ida5, C and D: pA7 transformants (7301B and 81110B), $\mathrm{E}$ : pA7/Tet $12 \mathrm{Chl}$ transformant $(8287 \mathrm{C})$. Acidic polypeptides to the right. Arrows indicate the position of actin; solid arrowheads, NAP; open arrowhead, the chimeric actin. the axoneme.

The axoneme of ida5 has been shown to contain NAP that appears as two spots with pIs of $\sim 5.6$ and $\sim 5.7$ in a two-dimensional electrophoresis pattern (10). To see whether NAP is present in these transformants, we carried out two-dimensional gel electrophoresis of the axoneme (Fig. 4). Unexpectedly, three (8114B, 81110B, $8113 \mathrm{C}$ ) of the six transformants with wild-type actin gene were found to contain NAP in addition to the conventional actin (Fig. 4D), while the rest of the transformants contain only the latter (Fig. 4C). Interestingly, NAP appears to be present in a greater amount in those transformants expressing smaller amounts of actin. In chimeric transformants, all were found to contain NAP in the axoneme in addition to the introduced chimeric actin (Fig. 4E).

\section{Recovery of inner-arm dynein and motility}

Recovery of inner-arm dynein in the axoneme of transformants was examined by several methods. First, SDSPAGE showed that at least one dynein heavy chain band that is absent from the ida5 axonemes was recovered in all transformants (Fig. 5). As a more quantitative analysis, we next extracted the axoneme of transformants with a high-salt concentration solution and examined the dynein composition by anion-exchange chromatography on a Mono Q column (Fig. 6). As reported previously (9), the elution pattern of the ida5 axonemal extract lacks four peaks corresponding to the inner-arm subspecies, $a, c, d$, and $e$. All these peaks were restored in the elution pattern of the transformants with the wild-type actin gene. The chimeric transformant also recovered the four subspecies, but the peaks were significantly smaller than those of wild-type or the actin transformants.

The axonemes of three transformants were also examined by electron microscopy. The inner arm of the wild-type axoneme in cross section appears as two projections, an outer-row (O-) projection and an innerrow (I-) projection $(8,9$, see also 16$)$. The I-projection, which has been shown to be absent in ida5 (Fig. 7C and D) (9), was restored by transformation with the wildtype actin gene (Fig. 7E and F). In chimeric actin transformant, however, the I-projection was only partially restored in some cross-section images of axoneme. The number of the I-projections in the axoneme of the chimeric transformant was counted in blind, and found to be fewer than that in the axoneme of wild type or of the transformant with the wild-type actin gene (Table I). These results are consistent with those of Western analysis (Fig. 3) and chromatography (Fig. 6).

Finally, the swimming velocities of the wild type, ida5 and transformants were compared. Wild-type cells swim at about $160 \mu \mathrm{m} / \mathrm{second}$ whereas the ida5 cells swim at about half the normal speed. All of the wild- 


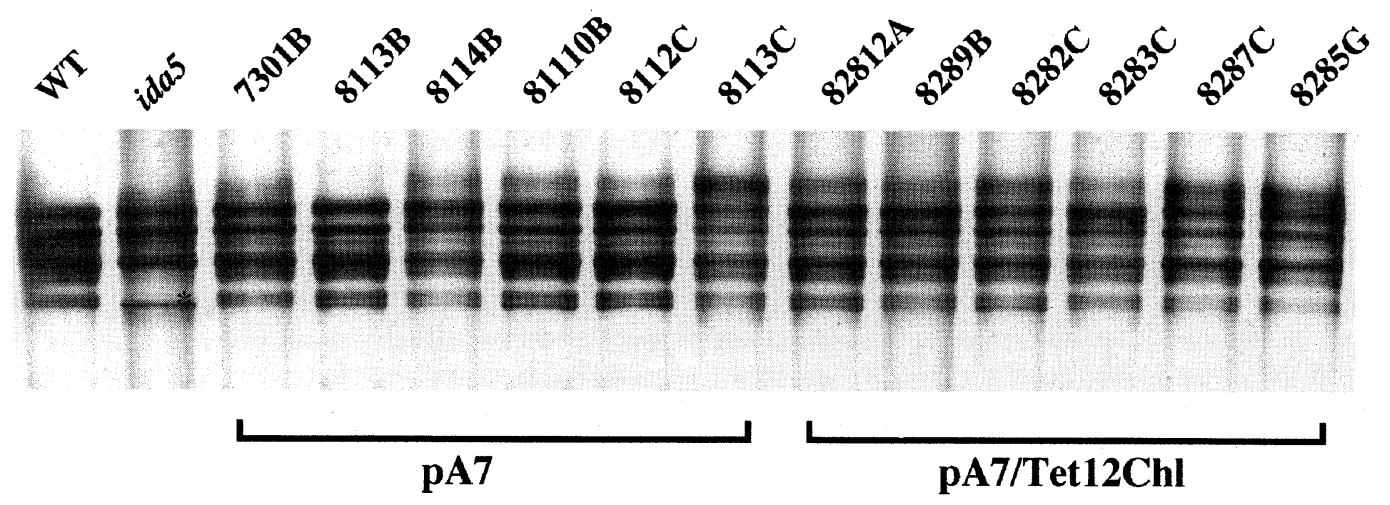

Fig. 5. SDS-PAGE patterns of the axonemes of wild type, ida5 and transformants. Part of a 3-5\% polyacrylamide gel stained with silver, showing dynein heavy-chain bands. Asterisk in ida5 pattern indicates the inner-arm band that are missing in ida5 axoneme. Note that the band indicated by asterisk is restored in all transformants.

type actin transformants recovered the motility almost to the normal level. On the other hand, all of the chimeric transformants recovered the motility to only about $80 \%$ of the normal level (Fig. 8).

\section{Production of the fertilization tubule}

A clear deficiency in ida5 is its inability to form the fertilization tubule that contains a core of F-actin bundles (10). In wild-type Chlamydomonas, this structure protrudes from the apical end of the plus-mating type gamete immediately after the onset of mating reaction, i.e.,

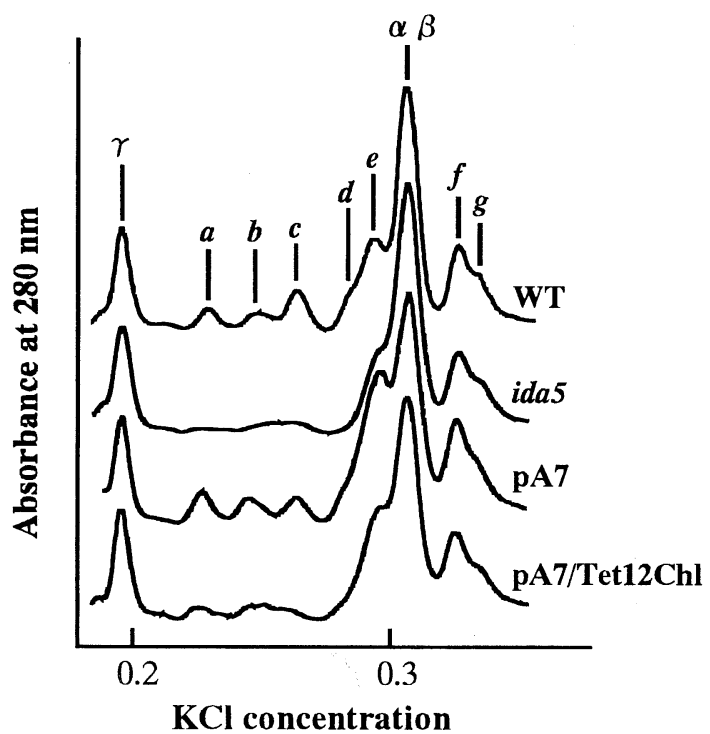

Fig. 6. Elution patterns of dyneins in high-pressure liquid chromatography. High-salt extracts of axonemes from wild type (WT), ida5, an actin transformants (pA7, 7301B) or a chimeric transformants (pA7/Tet12Chl, 8287C) were subjected to high-pressure liquid chromatography on a Mono Q column. Each peak of the outer-arm dyneins $(\alpha, \beta$, and $\gamma)$ and inner-arm dyneins $(a-g)$ are indicated on the top of the patterns. when the gametes of both mating types contact with each other by the flagella (3). The gametes can be induced to produce the fertilization tubule by treatment with dibutyryl-cAMP and IBMX (21). The plus-mating type gametes of the transformants were thus treated with these drugs and stained with BODIPY-phallacidin, a fluorescent probe for actin fibers. Observation with a fluorescence microscope revealed that five out of the six clones transformed with the wild-type actin gene produced fertilization tubules (Fig. 9), while none of the chimeric transformants produced them. The only wild-type actin transformant that did not produce the fertilization tubule is $81110 \mathrm{~B}$, the one that displayed a particularly low level of actin expression in Western analysis (Fig. 3A).

\section{DISCUSSION}

We have shown that the mutant phenotype of ida5 lack of inner-arm dynein and deficient fertilization tubule growth - can be rescued by transformation with the cloned actin gene. These results have established that those deficiencies are the direct consequences of the loss of conventional actin.

It is somewhat surprising that all of the wild-type actin transformants have recovered flagellar motility almost completely to the wild-type level despite the fact that their actin expression was lower than in wild type and variable from a transformant and another. This is in contrast with their ability to grow the fertilization tubule which apparently depended on the level of actin expression; for example, the transformant 81110B, which expresses a particularly low level of actin, did not produce the fertilization tubule in gametes. Thus Chlamydomonas appears to give high priority to actin as a subunit of inner-arm dynein. Transformants with a chimeric actin gene, in which an $\mathrm{N}$-terminal sequence was replaced by the divergent Tetrahymena actin se- 


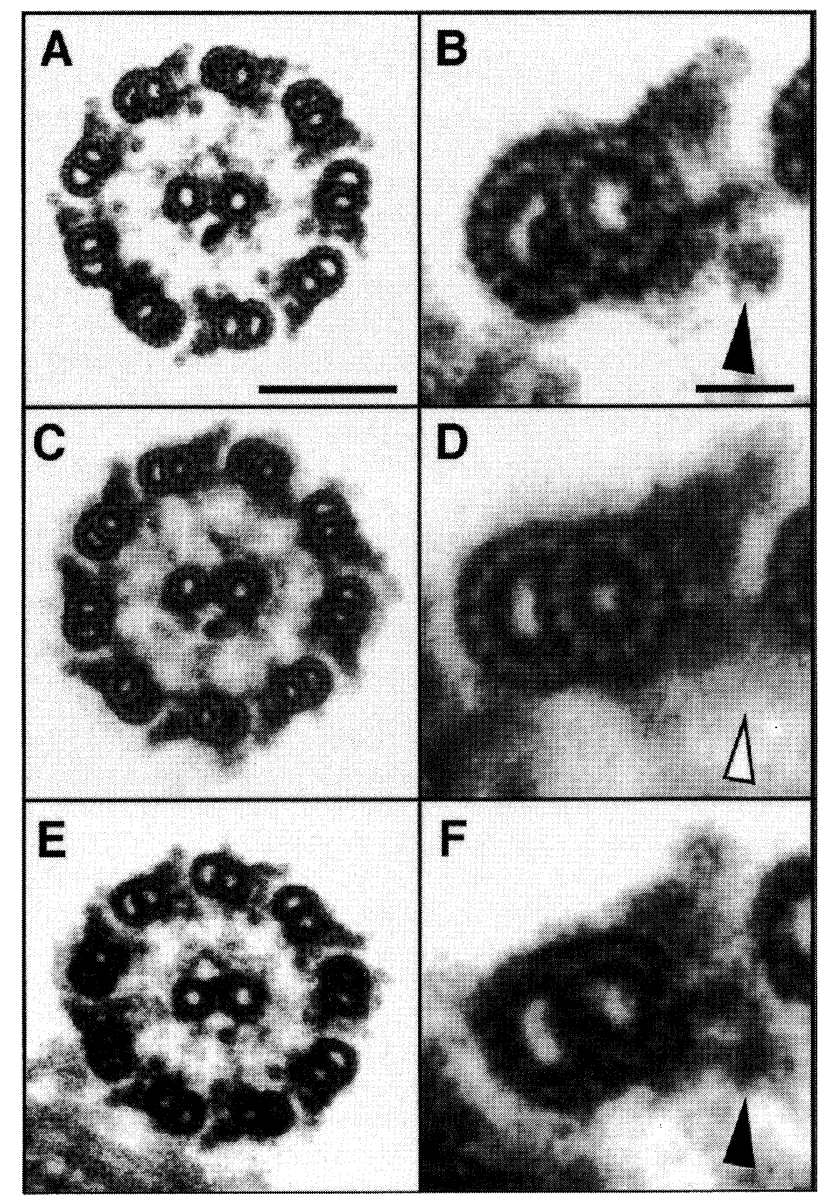

Fig. 7. Cross-section electron micrographs of axonemes (A, C, and E) and images of outer doublets (B, D, and F). A and B: wild type; C and D: ida5; E and F: actin transformant (7301B). Solid and open arrowheads indicate the position of the I-projection. Bars: $0.1 \mu \mathrm{m}$ (A, $\mathrm{C}$, and $\mathrm{E}$ ) and $0.02 \mu \mathrm{m}(\mathrm{B}, \mathrm{D}$, and F). Note that the image intensity of the inner row is very weak in idas (D), but it recovered in the actin transformants $(\mathrm{F})$.

Table I. NUMBER OF INNER-ROW (I-) AND OUTER-ROW (O-) PROJECTIONS OF INNER DYNEIN ARMS OBSERVED IN AN AXONEMAL CROSS SECTION.

\begin{tabular}{lccc}
\hline Strain & I-projection & O-projection & $\mathrm{n}$ \\
\hline wild type & $5.8 \pm 1.1$ & $5.8 \pm 0.9$ & 10 \\
ida5 & $0.3 \pm 0.5$ & $5.9 \pm 0.6$ & 8 \\
7301B (actin transformant) & $5.5 \pm 1.2$ & $5.6 \pm 1.2$ & 8 \\
8287C (chimeric transformant) & $3.9 \pm 1.7$ & $5.7 \pm 1.2$ & 10 \\
\hline
\end{tabular}

The number of I- and O-projections that were clearly identified in an axonemal cross-section electron micrograph was counted. The average \pm standard deviation in 8-10 axonemes is shown for each specimen. n: number of axonemes counted. The number of I-projections in $8287 \mathrm{C}$ axoneme is significantly smaller than that in $7301 \mathrm{~B}$ axoneme $(\mathrm{P}<0.05)$

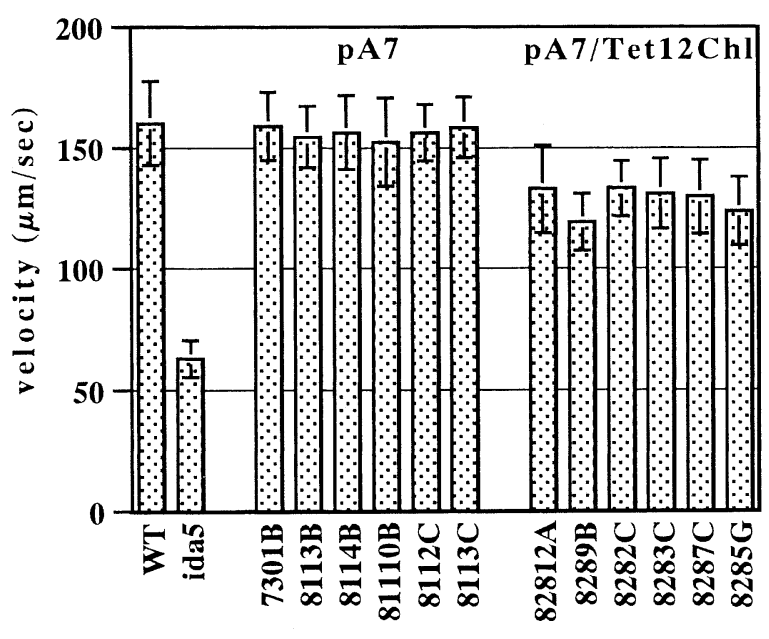

Fig. 8. Swimming velocities of the wild type, ida5 and transformants. The average and standard deviation (bar) of swimming velocities measured in 25 cells are shown for each transformant. The average velocity in any one of the six actin transformants (e.g., 81110B) and that in any one of the six chimeric transformants (e.g., 82812A) are significantly different $(P<0.001)$.

quence (6), also recovered dynein and motility to about $70-80 \%$ of the wild-type level, but not the fertilization tubule growth in gametes. The incomplete recovery of dynein and lack of fertilization tubule growth are probably due to their extremely low levels of actin expression, as suggested by the Western analysis using whole cell lysates. Although the exact reason for the poor expression of chimeric actin is not known, we speculate that an important reason is the great difference in codon usage in Tetrahymena and Chlamydomo-
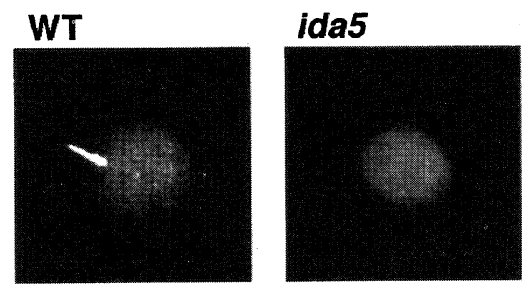

\section{$7301 B$}
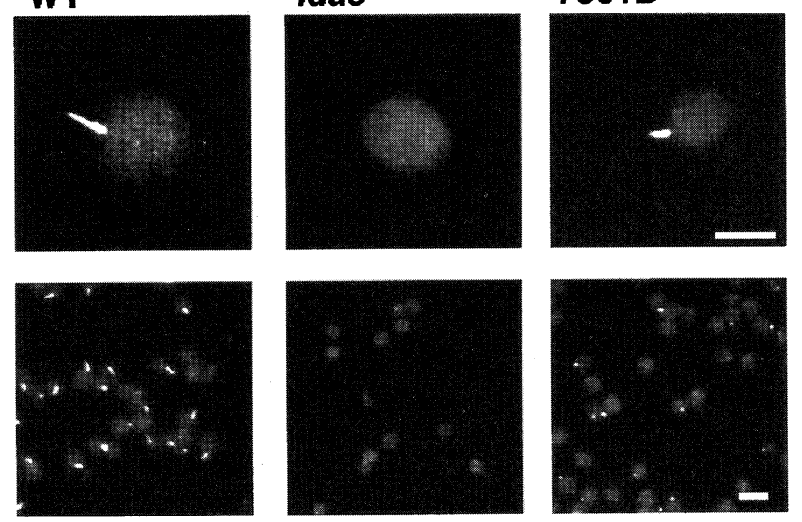

Fig. 9. Fluorescence micrographs of the fertilization tubule visualized with BODIPY-phallacidin. WT: wild type, 7301B: a transformant with pA7. Bars: $5 \mu \mathrm{m}$ (upper panel) and $10 \mu \mathrm{m}$ (lower panel). The growth of fertilization tubules was induced by incubating gametic cells of the plus-mating type in the presence of $1 \mathrm{mM}$ IBMX and 15 mM dibutyryl cAMP for 1 hour (21). 
nas. Because of the very low level of expression of the chimeric actin, the present study did not allow us to determine whether the altered 12 amino-acid sequence in the chimeric actin has any specific effects on the actin function in Chlamydomonas. However, we can at least say that the actin with the altered $\mathrm{N}$-terminal sequence can function as the subunit of inner-arm dyneins, since the chimeric actin expressed in small amounts resulted in partial recovery of inner-arm dyneins. Hence the $\mathrm{N}$ terminal sequence of actin may not be critical for the function of actin as a dynein subunit.

The axonemes from three out of the six wild-type actin transformants contained NAP in addition to the actin expressed from the introduced gene(s) (Fig. 4). These three transformants were among those that displayed weak actin expression. Together with the finding that the axonemes of all of the chimeric transformants contain NAP, these findings indicate that NAP occurs in those transformants that do not produce suffi cient amounts of actin. NAP has been shown to be associated with two subspecies of inner-arm dynein, called $b$ and $g$, in the axoneme of $i d a 5$. However, in the wild-type axoneme, actin, but not NAP, is associated with these and other inner-arm subspecies; it has thus been speculated that NAP can substitute for actin as subunits of subspecies $b$ and $g$, but not as subunits of other inner arms (10). It is conceivable that, in those transformants with low levels of actin expression, the two inner-arm subspecies $b$ and $g$ contain NAP while other subspecies contain actin.

Rescue of mutant phenotypes by transformation with cloned wild-type genes is widely used in Chlamydomonas to select transformants $(1,12)$, and to confirm or identify the mutated gene $(2,17,25,30)$. Except for rare instances $(19,26)$, most nuclear transformation events in Chlamydomonas have been shown to take place as non-homologous recombination; i.e., exogenous genes are incorporated into loci different from those of the original, mutated genes. In the present study, we also confirmed that, in at least two transformants, the incorporated actin genes do not link with the original actin locus, since genetic crosses of the transformants with arg2 yielded daughter cells that display the slow-swimming phenotype of ida5. The fact that the actin gene incorporated into non-homologous sites produced sufficient amount of actin, at least in some transformants, indicates that the plasmid we used contained a full set of mucleotide sequences required for actin expression. The availability of functional actin clones and a null mutant of conventional actin, ida5, should provide a powerful experimental system with which to study the molecular mechanisms of actin function by means of in vitro mutagenesis.

\section{REFERENCES}

1. Debuchy, R., Purton, S., and Rochaix, J.D. 1989. The argininosuccinate lyase gene of Chlamydomonas reinhardtii: an important tool for nuclear transformation and for correlating the genetic and molecular maps of the ARG7 locus. EMBO J., 8: 2803-2809.

2. Diener, D.R., Curry, A.M., Johnson, K.A., Williams, B.D., Lefebvre, P.A., Kindle, K.L., and Rosenbaum, J.L. 1990. Rescue of a paralyzed-flagella mutant of Chlamydomonas by transformation. Proc. Natl. Acad. Sci., 87: 5739-5743.

3. Friedman, I., Colwin, A.L., and Colwin, L.H. 1968. Finestructural aspects of fertilization in Chlamydomonas reinhardtii. J. Cell Sci., 3: 115-128.

4. Gorman, D.S. and Levine, R.P. 1965. Cytochrome f and plastocyanin: their sequence in the photosynthetic electron transport chain of Chlamydomonas reinhardtii. Proc. Natl. Acad. Sci. USA, 54: 1665-1669.

5. Harris, E.H. 1989. The Chlamydomonas Sourcebook. Academic Press, San Diego. 780 pp.

6. Hirono, M., Endoh, H., Okada, N., Numata, O., and WATANABE, Y. 1987. Tetrahymena actin: Cloning and sequencing of the Tetrahymena actin gene and identification of its gene product. J. Mol. Biol., 194: 181-192.

7. Kagami, O. and KamiYa, R. 1992. Translocation and rotation of microtubules caused by multiple species of Chlamydomonas inner-arm dynein. J. Cell Sci., 103: 653-664.

8. Kamiya, R., Kurimoto, E., and Muto, E. 1991. Two types of Chlamydomonas flagellar mutants missing different components of inner-arm dynein. J. Cell Biol., 112: 441-447.

9. Kato, T., Kagami, O., YAGI, T., and KamiYA, R. 1993. Isolation of two species of Chlamydomonas reinhardtii flagellar mutants, ida5 and ida6, that lack a newly identified heavy chain of the inner dynein arm. Cell Struct. Funct., 18: 371-377.

10. Kato-Minoura, T., Hirono, M., and Kamiya, R. 1997. Chlamydomonas inner-arm dynein mutant, ida5, has a mutation in an actin-encoding gene. J. Cell Biol., 137: 649-656.

11. KindLE, K.L. 1990. High-frequency nuclear transformation of Chlamydomonas reinhardtii. Proc. Natl. Acad. Sci. USA, 87: 1228-1232.

12. Kindle, K.L., Schnell, R.A., Fernandez, E., and LefebVre, P.A. 1989. Stable nuclear transformation of Chlamydomonas using the Chlamydomonas gene for nitrate reductase. J. Cell Biol., 109: 2589-2601.

13. King, S.M., Marchese-Ragona, S.P., Parker, S.K., and DETRICH, H.W.3rd. 1997. Inner and outer arm axonemal dyneins from the Antarctic rockcod Notothenia coriiceps. Biochemistry, 36: 306-314.

14. LAEMMLI, U.K. 1970. Cleavage of structural proteins during the assembly of the head of bacteriophage T4. Nature, 227: 680685.

15. Lee, V.D., Finstad, S.L., and Huang, B. 1997. Cloning and characterization of a gene encoding an actin-related protein in Chlamydomonas. Gene, 197: 153-159.

16. Mastronarde, D.N., O'Toole, E.T., McDonald, K.L., McIntosh, J.R., and Porter, M.E. 1992. Arrangement of inner dynein arms in wild-type and mutant flagella of Chlamydomonas. J. Cell Biol., 118: 1145-1162.

17. Mitchell, D.R. and KANG, Y. 1991. Identification of oda6 as a Chlamydomonas dynein mutant by rescue with the wild-type gene. J. Cell Biol., 113: 835-842.

18. Muto, E., Edamatsu, M., Hirono, M., and Kamiya, R. 1994. 
Immunological detection of actin in the $14 \mathrm{~S}$ ciliary dynein of Tetrahymena. FEBS Lett., 343: 173-177.

19. Nelson, J.A. and Lefebvre, P.A. 1995. Targeted disruption of the NIT8 gene in Chlamydomonas reinhardtii. Mol. Cell Biol., 15: 5762-5769.

20. Olmsted, J.B. 1981. Affinity purification of antibodies from diazotized paper blots of heterogeneous protein samples. $J$. Biol. Chem., 256: 11955-11957.

21. Pasquale, S.M. and Goodenough, U.W. 1987. Cyclic AMP functions as a primary sexual signal in gametes of Chlamydomonas reinhardtii. J. Cell Biol., 105: 2279-2292.

22. Pfister, K.K., FAy, R.B., and Witman, G.B. 1982. Purification and polypeptide composition of dynein ATPases from Chlamydomonas flagella. Cell Motil., 2: 525-547.

23. PIPERno, G. and LUCK, D.J.L. 1979. An actin-like protein is a component of axonemes from Chlamydomonas flagella. $J$. Biol. Chem., 254: 2187-2190.

24. Pratt, M.M. 1986. Homology of egg and flagellar dynein. Comparison of ATP binding sites and primary structure. $J$. Biol. Chem., 261: 956-964.

25. SMith, F.E. and Lefebvre, P.A. 1996. PF16 encodes a protein with armadillo repeats and localizes to a single microtubule of the central apparatus in Chlamydomonas flagella. J. Cell Biol., 132: 359-370.

26. Sodeinde, O.A. and KIndLE, K.L. 1993. Homologous recombination in the nuclear genome of Chlamydomonas reinhardtii. Proc. Natl. Acad. Sci. USA, 90: 9199-9203.
27. StriJkert, P.J., Loppes, R., and SusSenbaCh, J.S. 1973. The actual biochemical block in the arg-2 mutant of Chlamydomonas reinhardtii. Biochemical Genetics, 8: 239-248.

28. Sugase, Y., Hirono, M., Kindle, K.L., and KamiYa, R. 1996. Cloning and characterization of actin-encoding gene of Chlamydomonas reinhardtii. Gene, 168: 117-121.

29. Sutoh, K., Ando, M., and Toyoshima, Y.Y. 1991. Site-directed mutations of Dictyostelium actin: disruption of a negative charge cluster at the $\mathrm{N}$ terminus. Proc. Natl. Acad. Sci. $U S A$, 88: 7711-7714.

30. TAM, L.-W. and Lefebvre, P.A. 1993. Cloning of flagellar genes in Chlamydomonas reinhardtii by DNA insertional mutagenesis. Genetics, 135: 375-384.

31. Towbin, H., Staehelin, T., and Gordon, J. 1979. Electrophoretic transfer of proteins from polyacrylamide gels to nitrocellulose sheets: procedure and some applications. Proc. Natl. Acad. Sci. USA, 76: 4350-4354.

32. Weeks, D.P., Beerman, N., and Griffith, O.M. 1986. A small-scale five-hour procedure for isolating multiple samples of CsCl-purified DNA: application to isolations from mammalian, insect, higher plant, algal, yeast, and bacterial sources. Anal. Biochem., 152: 376-385.

33. Witman, G.B. 1986. Isolation of Chlamydomonas flagella and flagellar axonemes. Methods Enzymol., 134: 280-290.

(Received for publication, June 26, 1998

and in revised nform, August 5, 1998) 